\title{
A NEW METHOD OF ANALYZING a-RAY PHOTOGRAPHS
}

\author{
By L. F. Curtiss
}

\section{ABSTRACT}

A design for a double camera for photographing $\alpha$-ray tracks is described. The two cameras permit mutually perpendicular views of the tracks to be taken while at the same time the two object planes coincide throughout. This makes it possible to photograph more tracks at a single exposure than would be possible if the object planes were, as usual, inclined at $90^{\circ}$ to each other. The two cameras are rigidly connected so that a new method of analyzing the negatives may be used. This method consists in projecting the negatives back on the object plane and orienting a photographic plate so that a single full-size reproduction of the original track is obtained in the plane in which it originally occurred.

Investigations of $\alpha$-ray collisions with atomic nuclei by means of the Wilson cloud chamber require a method of photographing the tracks which will permit a determination of the angles between the paths of the various particles in whatever plane they may occur. The method most commonly used for this purpose is the arrangement described by Shimizu ${ }^{1}$ which employs a system of mirrors so that two mutually perpendicular views of the tracks are registered by a single lens on the same portion of a cinematograph film. Blackett ${ }^{2}$ has published mathematical treatments of the problem of analyzing the photographs thus obtained to determine the actual angles and length of path. While this method at first sight seems to be economical of film, which is an important point where many thousands of photographs are necessary, it is not really so. Since the two object planes are inclined $90^{\circ}$ to each other, only a small region of the cloud chamber is in sharp focus for both views and, hence, only a few, not more than a dozen, tracks may be photographed at each exposure, and even then all portions of the tracks are rarely in good focus on both negatives.

Blackett ${ }^{3}$ has described a method of eliminating this difficulty. Two cameras with two separate lenses are required. The focal plane of one camera is superposed on that of the other so that the whole cross section of the chamber is sharply in focus in both views. It is then possible to photograph upwards of 50 tracks at each exposure and have all sharply focussed in both views. Thus, although twice as much film is used for a photograph, four or five times as many tracks are obtained-all in good focus.

As Blackett has shown, there is a limit to the number of tracks which will be resolved in both views, which will reduce considerably the optimum number of tracks. However, for some purposes it is

1 Shimizu, T., Proc. Roy. Soc., 99, p. 425; 1921.

2 Blackett, P. M. S., Proc. Roy. Soc., 102, p. 294; 1922; 103, p. 62; 1923.

B Blackett, P. M. S., Proc. Roy. Soc., 123, p. 613; 1929. $101062^{\circ}-30-5$ 
permissible to exceed considerably the optimum numbers which Blackett gives and still obtain reasonably satisfactory results.

The method of analysis developed by Blackett may still be used to determine the angles between the tracks, since the geometry of the system is not changed. However, there is a more simple and direct way of obtaining this information, for suggesting which I am indebted to Dr. A. v. Hippel, of the University of Jena. This paper describes a camera specially constructed to make use of this suggestion, the details of which will become obvious from the description of the camera and its use.

The optical system is illustrated by the diagram shown in Figure 1. Each of the two cameras, $C_{1}$ and $C_{2}$, has its own lens and film. The cameras are inclined $45^{\circ}$ to the horizontal. This arrangement alone would still leave the object planes inclined $90^{\circ}$ to each other. These planes may be tilted, however, until they are superposed by inclining the axis of the lens of each camera as shown so that the perpendicular to the axis $\left(a_{1}, b_{1}\right)\left(a_{2}, b_{2}\right)$ of each lens passes through the points of intersection, $a_{1}, a_{2}$, of a line through the center of the

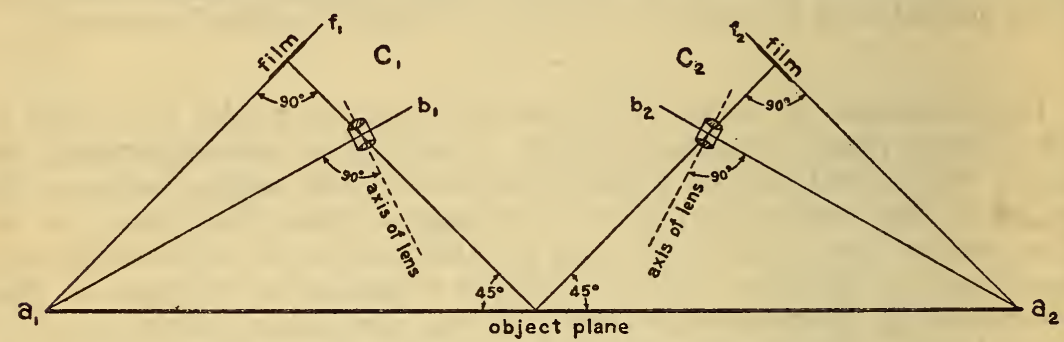

FIgURE 1.-Optical system of double camera

object plane, $a_{1}, a_{2}$, and lines $\left(a_{1}, f_{1}\right)\left(a_{2}, f_{2}\right)$ through the centers of the image planes. ${ }^{4}$ This involves a rectilinear distortion of the object in the images. As will be seen, this is automatically taken care of by the method of analyzing the photographs which is to be described.

This method of analysis consists merely in replacing the developed negatives in the film holders of the camera in the exact position in which they were when the photographs were taken and projecting the two images back on to the original object plane. A thin, white, translucent screen is then adjusted and rotated until the two views coincide in all respects. We then have very accurately an exact reproduction geometrically of the original track in the plane in which it was originally formed. Thus this procedure in one operation (1) locates the plane in which the track was formed, (2) corrects the rectilinear distortion in the photographic negative arising from tilting the axis of the lens with respect to the perpendicular to the negative, (3) enlarges the image to full size. Since the same lenses are used for projection and the negatives are in the position which they had when the exposure was made, many difficulties which might arise from unequal adjustment of the parts of the cameras are also eliminated. It is now only necessary to replace the white screen by a photographic plate to secure a permanent record of each track which then may be measured at leisure. This method of analysis also

4 Glazebrook, Dict. of Applied Phys., 4, p. 400. 
B. S. Journal of Research, RPI7!

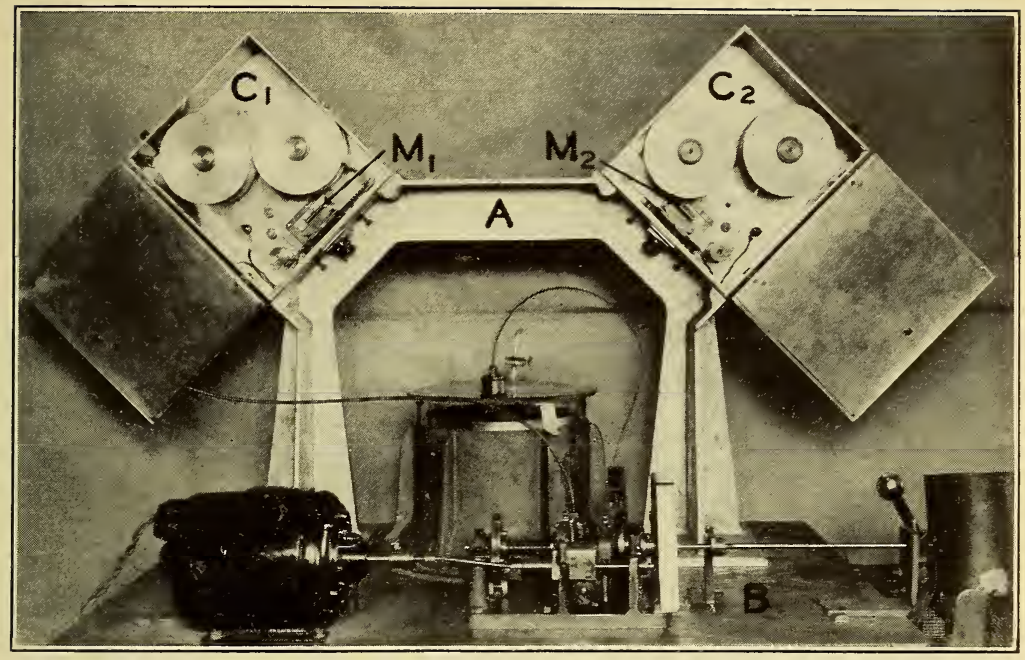

Figure 2.-Camera in position above expansion chamber

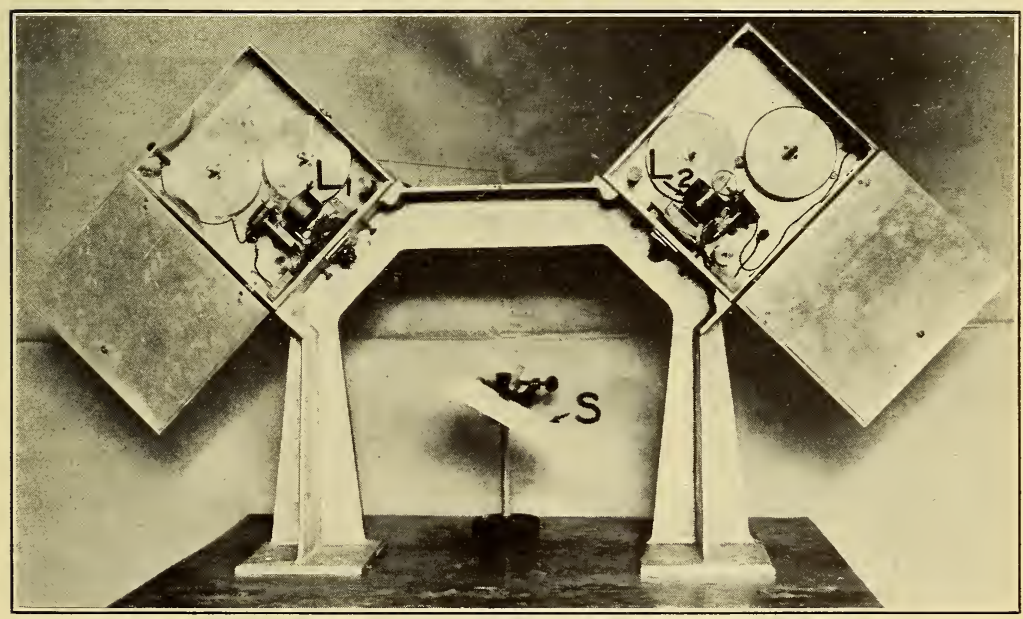

FIGURE 3.-Arrangement of camera for projecting negatives 
B. S. Journal of Research, RPI7!

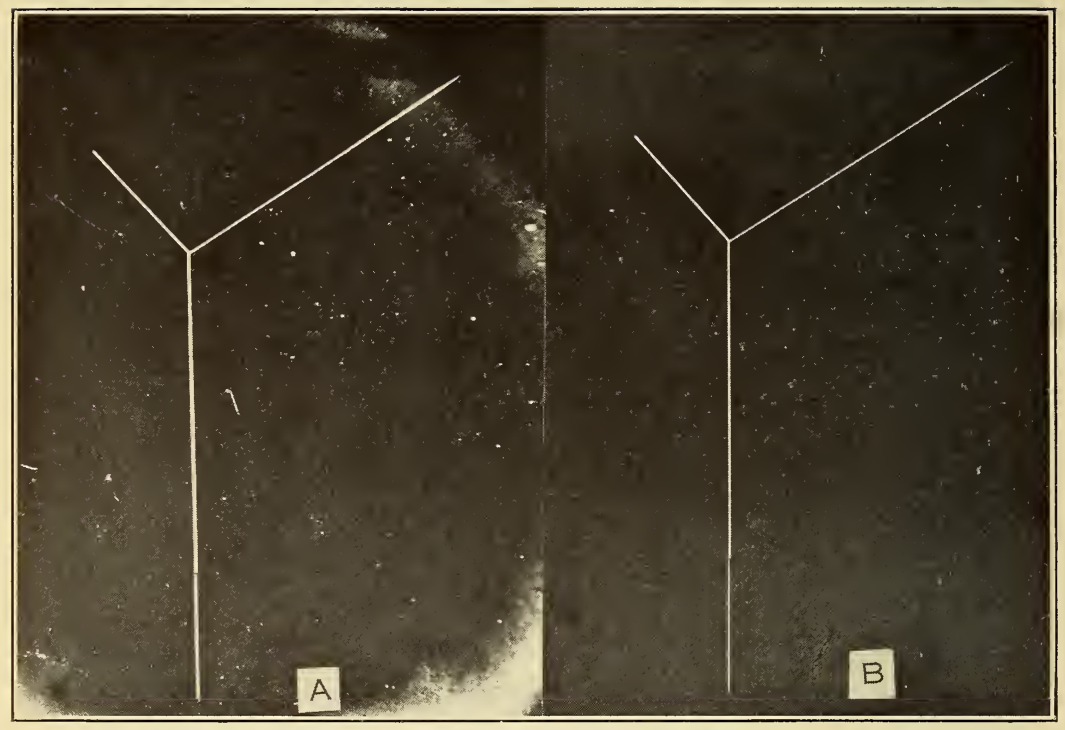

Figure 4.-Precision of reproduction 
avoids difficulties which are encountered in the mathematical analysis when any of the angles are near $90^{\circ}$.

In order to make use of this method the two cameras must be mounted on a single casting so that they may be removed from the expansion chamber when one wishes to use them for reproducing images of tracks. Figure 2 shows how this has been accomplished. A single aluminum casting, $A$, forms a support for the lenses and film holders of the two cameras, $C_{1}$ and $C_{2}$, so that there can be no change of alignment. The casting is held down to the base of the expansion chamber, $B$, by screws which may be removed. The cameras may then be placed in a convenient position for projection. Figure 3 shows the camera fitted with lights for projecting negatives, and illustrates the adjustment of the screen. To facilitate replacing the negatives in the exact position which the film occupied when the exposure was made, the electromagnets $M_{1}$ and $M_{2}$ (fig. 2) are arranged to push a needle point through the film at the instant of exposure. Since the guides fit the film snugly the film can be replaced by adjusting it until the needle point passes into the hole in the film made at the time of exposure.

In Figure 3 the cameras are shown removed from the base of the expansion apparatus and equipped for projection of negatives. Two automobile headlight bulbs, fitted with condensing lenses, $L_{1}$ and $L_{2}$, are required for illuminating the negatives. These will fit readily into the camera when the film spools are removed as shown. The images of the negatives are thrown on the screen $S$, which is adjusted until a satisfactory position has been found. This is then replaced by a photographic plate and a permanent record of the track obtained.

The adjustment of the position of the screen is very sensitive, even slight displacements causing some portion of the image to appear double. Repeated trials with model tracks have shown the method to be sufficiently accurate for the purpose. Figure 4 shows $(a)$ an image obtained by projecting two photographs of a model track on a photographic plate and $(b)$ the model track (drawn on white cardboard) itself. In this case the plane of the fork was inclined about $30^{\circ}$ to the horizontal. The two are identical within ordinary limits of error. Trials have also been made with model tracks made with silk thread placed in various positions with similar results.

Washington, December 21, 1929. 